\title{
Gestion de la chenille mineuse de l'épi du mil par des lâchers du parasitoïde Habrobracon hebetor Say au Niger
}

\author{
Laouali Amadou ${ }^{1}$, Ibrahim B. Baoua ${ }^{2, *}$, Malick N. Ba ${ }^{3}$, Bettina Haussmann ${ }^{4}$ et Mariama Altiné \\ ${ }^{1}$ Institut national de la recherche agronomique du Niger (INRAN), CERRA de Maradi, BP 240, Maradi, Niger \\ 2 Université DanDicko Dankoulodo de Maradi, BP 465, Maradi, Niger \\ ${ }^{3}$ International Crops Research Institute for the Semi-Arid Tropics (ICRISAT), Niamey, Niger \\ ${ }^{4}$ University of Hohenheim, Institute of Plant Breeding, Seeds Science and Population Genetics, Stuttgart, Germany \\ ${ }^{5}$ Programme de la productivité agricole en Afrique de l'Ouest (PPAAO/WAAPP), Niamey, Niger
}

\begin{abstract}
Résumé - La chenille mineuse de l'épi du mil, Heliocheilus albipunctella De Joannis, est l'un des principaux ravageurs du mil en zone sahélienne. En 2015 et 2016, une démarche de lutte biologique de grande envergure a été initiée par des lâchers du parasitoïde antagoniste ectolarvaire Habrobracon hebetor Say dans les cinq régions du Niger. Des équipes incluant les organisations de producteurs ont été mises en place pour la sensibilisation des agriculteurs, l'identification des zones d'infestation du ravageur et la mise en place de 9177 sacs de lâcher. Les surfaces couvertes ont été estimées à 1417680 ha en 2015 et 1521330 ha en 2016 soit $21 \%$ des surfaces en mil dans les régions concernées. Les évaluations menées au cours des deux années ont permis de noter une augmentation des taux de parasitisme de la chenille mineuse de 168 à 1398 \% dans les villages de lâcher par rapport aux villages témoins. L'enquête sur la perception a révélé une appréciation positive de $77 \%$ des répondants. Ils ont estimé la mortalité moyenne des larves du ravageur à $76 \%$ et les gains de rendement à $50 \%$.
\end{abstract}

Mots clés : Pennisetum glaucum / Heliocheilus albipunctella / Habrobracon hebetor / enquête / perception

Abstract - Management of the pearl millet head miner through releases of the parasitoid wasp Habrobracon hebetor Say in Niger. Millet head miner Heliocheilus albipunctella De Joannis is one of the main insect pests of millet in the Sahel. In 2015 and 2016, a large-scale biological process was initiated with the parasitoid Habrobracon hebetor Say released in five regions of Niger. Regional teams, including farmers' organizations, were set up for promoting producers' awareness, identifying infested areas and placing 9177 release bags. The surface covered was estimated to be 1417680 ha in 2015 and 1521330 ha in 2016, about $21 \%$ of the millet cropped area in the regions concerned. Evaluations conducted during two years showed an increase of $168 \%$ to $1398 \%$ for the parasitism rate on MHM larvae in the villages where parasitoids were released compared to control villages. Survey about farmers' perception revealed that the operation is beneficial for more than $77 \%$ of respondents. They estimated an average $76 \%$ larvae mortality rate and a $50 \%$ increase in millet yield due to the parasitoid release.

Keywords: Millet / Heliocheilus albipunctella / biological control / Habrobracon hebetor / survey / perception

\section{Introduction}

Le mil, Pennisetum glaucum (L.) R. Br., est l'une des céréales les plus cultivées dans les régions arides et semi-arides (Sehgal et al., 2012). Au Niger, il est utilisé par $97 \%$ des ménages et représente $23 \%$ de la consommation alimentaire (INS, 2011).

\footnotetext{
*Auteur de correspondance : baoua.ibrahim@gmail.com
}

Cependant, la culture du mil est, au Sahel ouest-africain, confrontée depuis les grandes sécheresses des années 1974 aux ravages causés par la chenille Mineuse de l'Épi du Mil (MEM) Heliocheilus albipunctella De Joannis (Lepidoptera, Noctuidae) [Vercambre, 1978]. Ce lépidoptère qui se développe sur l'organe de fructification du mil (Gahukar, 1984) peut causer des pertes de rendement en grain de $60 \%$ en années de fortes pullulations.

Les premières études pionnières de la lutte biologique ont débuté dans les années 1980 avec l'inventaire des prédateurs, 
parasitoïdes et parasites autochtones de la MEM (Bhatnagar, 1987; N'Doye et al., 1984). Des lâchers d'inoculation du parasitoïde Habrobracon hebetor Say (Hymenoptera : Braconidae) en champ ont été envisagés au Sénégal dès 1985 (Bhatnagar, 1989).

Vu la nécessité de couvrir de nombreux villages, il a été mis au point un dispositif dont la dernière version consiste en un sac de jute de $10 \mathrm{~cm} \times 7 \mathrm{~cm}$ qui contient $80 \mathrm{~g}$ de mil, 25 larves de Corcyra cephalonica Stainton (Lepidoptera: Pyralidae) et deux femelles fécondées de $H$. hebetor (Baoua et al., 2014). Ce système libère en moyenne 57 à 71 parasitoïdes, huit jours après sa préparation et pendant une période de deux semaines. Ce programme, financé par la fondation McKnight (http:// mcknight.ccrp.cornell.edu/projects/waf_cop/WAF_09-036/ 09-036_project.html), a permis l'expérimentation des lâchers dans plus de 400 villages du Niger, du Burkina Faso et du Mali (Payne et al., 2011).

Nous partageons et discutons ici une expérience de diffusion à large échelle de cette technologie dans les cinq régions agricoles du Niger.

\section{Matériels et méthode}

L'étude a été conduite dans cinq régions agricoles du Niger situées entre 1 et $12^{\circ}$ de latitude est et 12 et $16^{\circ}$ de longitude nord, dans un climat est de type sahélien et sahélo-soudanien. Ces régions totalisent $97 \%$ des surfaces emblavées en mil au Niger ( 7170000 ha) et $93 \%$ des 12200 villages agricoles du pays (MA, 2015).

La démarche de mise en place a suivi plusieurs étapes:

- la formation de tous les acteurs à la lutte biologique contre la MEM, avec comme thématiques: la biologie de la MEM, la lutte biologique et ses modalités de mise en œuvre, l'évaluation d'un programme de lutte;

- la mise en place d'équipes régionales d'intervention composées de cadres des services techniques du ministère de l'Agriculture et d'animateurs des organisations paysannes pour:

- identifier des zones potentielles d'attaque de la MEM en repérant les zones de semis hâtif du mil, présentant des sols dunaires et des antécédents de dégâts de la MEM,

- confirmer les zones d'infestation avec le déplacement des équipes pour repérer les pontes du ravageur dans les zones ciblées,

- mettre en place le dispositif de lutte biologique au niveau des villages concernés par les infestations;

- la surface couverte par les lâchers de $H$. hebetor a été déterminée de la façon suivante : un dispositif de 15 sacs de lâcher placés dans chaque village couvre au moins un rayon de $5 \mathrm{~km}$ soit 7850 ha (Baoua et al., 2014; Payne et al., 2011). Il a été considéré qu'au moins $60 \%$ des surfaces autour des villages sont semées en mil;

- pour chacune des années, l'évaluation de l'opération a été effectuée par les chercheurs avec l'appui des animateurs des organisations paysannes. Elle a concerné 13 villages par région: dix villages ayant bénéficié des lâchers de $H$. hebetor et trois villages concernés par les attaques de la MEM, mais pour lesquels il n'y a pas eu d'intervention (témoins). Pour chacun des villages, 400 épis ont été prélevés de façon aléatoire dans quatre champs. Il a été noté pour chaque épi, le nombre total de mines et de celles portant des dépouilles de larves de la MEM parasitées par H. hebetor. Les larves parasitées sont identifiables par la présence des cocons d'émergence du parasitoïde à leur côté.

- Ces données ont permis de calculer le pourcentage des épis infestés par la MEM et la proportion des épis infestés portant des larves de la MEM parasitées par $H$. hebetor;

- une enquête sur la perception des producteurs a été conduite en 2015 dans 15 villages choisis aléatoirement dans chacune des régions. Un questionnaire a été administré à 732 producteurs, dont $5 \%$ de femmes. Il s'agit pour la plupart de producteurs de mil impliqués dans les activités de lutte biologique. Les questions ont porté sur la biologie et les mortalités de la MEM, le rayon d'action du dispositif et l'estimation des gains de rendement dans leurs champs après les lâchers du parasitoïde. Le logiciel ODK (Open Data Kit) (https://opendatakit.org/) a été utilisé pour la collecte des données.

Analyses statistiques : L'analyse de variance a été effectuée pour comparer les niveaux d'infestation entre les régions. Le test $t$ de Student Newman Keuls a permis de comparer les taux de parasitisme des larves de la MEM entre les villages de lâcher et les villages témoins. Ces analyses ont été effectuées avec le logiciel IBM SPSS Statistics 20. Le logiciel Arcview 9.3 a été utilisé pour produire une carte des points de lâchers de $H$. hebetor.

\section{Résultats}

En 2015, 545 villages à risque ont été identifiés, soit 4360000 ha de mil menacés par la MEM, et 3126 villages en 2016, soit 7879400 ha potentiellement infestés par le ravageur. Les besoins exprimés en sacs de lâcher de $H$. hebetor étaient de 8175 unités en 2015 et 5550 unités en 2016 . Les visites sur le terrain ont permis de confirmer les infestations de la MEM dans $55 \%$ des villages à risque de 2015 et $10 \%$ de ceux de 2016.

La sensibilisation a concerné plus de 7500 producteurs en 2015 et 22000 producteurs en 2016; respectivement $15 \%$ et $21 \%$ étaient des femmes. Quatre mille cinq cent quinze sacs ont été placés dans 301 villages en 2015, et 4662 sacs dans 323 villages en 2016. Pour les cinq régions, au cours des deux années, les opérations de lutte biologique ont couvert 574 villages, dont $9 \%$ ont été concernés par les lâchers du parasitoïde pendant les deux années consécutives (Fig. 1). Les surfaces cultivées en mil couvertes par le parasitoïde ont été estimées à 1417680 ha en 2015 et 1521330 ha en 2016.

Les évaluations dans les villages ont permis de noter des taux d'infestation des épis qui diffèrent d'une région à une autre (Tab. 1). Pour les deux années, le plus faible taux d'épis infestés a été enregistré dans la région de Maradi (plutôt au Sud) et les plus élevés dans les régions de Tahoua et Tillabéry (plutôt au Nord).

La proportion des mines portant des larves de la MEM parasitées par $H$. hebetor a varié entre les villages de lâcher et 

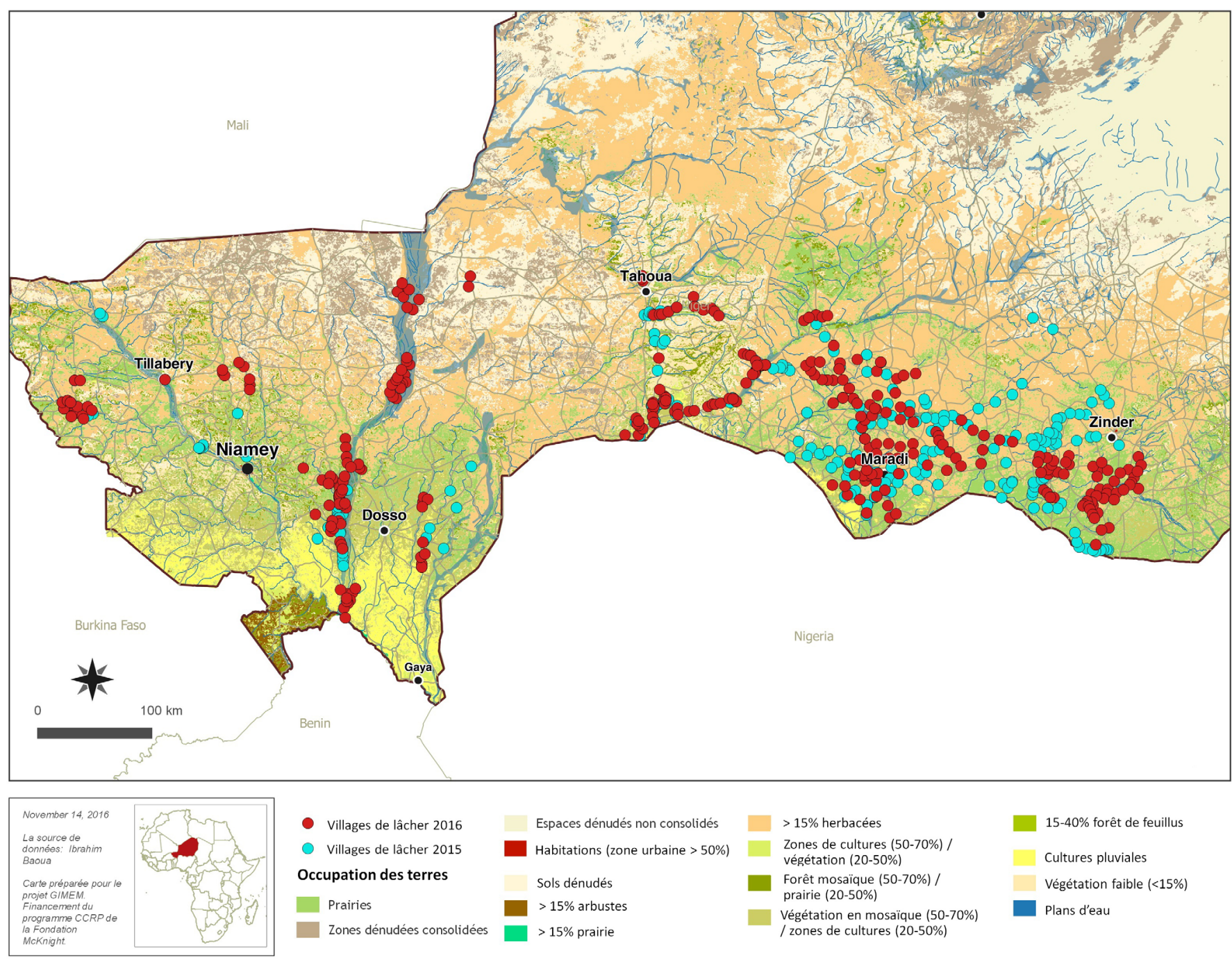

15-40\% forêt de feuillus

Cultures pluviales Végétation faible $(<15 \%)$ Plans d'eau

Fig. 1. Cartographie des villages de lâcher du parasitoïde Habrobracon hebetor Say en 2015 et 2016.

Fig. 1. Map of releases of the parasitoid wasp Habrobracon hebetor Say in 2015 and 2016.

Tableau 1. Taux moyens d'infestation de la culture du mil par le lépidoptère Heliocheilus albipunctella De Joannis en 2015 et 2016. Table 1. Average infestation of pearl millet crops by lepidoptera Heliocheilus albipunctella De Joannis in 2015 et 2016.

\begin{tabular}{|c|c|c|c|c|}
\hline \multirow[b]{2}{*}{ Régions } & \multicolumn{4}{|c|}{ Proportion des épis de mil infestés par la MEM (\%) } \\
\hline & $n$ & 2015 & $n$ & 2016 \\
\hline Dosso & 64 & $19,68 \pm 2,45 \mathrm{ab}$ & 45 & $10,80 \pm 0,70 \mathrm{a}$ \\
\hline Tahoua & 77 & $31,80 \pm 3,71 b$ & 46 & $19,07 \pm 1,81 b$ \\
\hline Tillabéry & 51 & $31,82 \pm 0,84 b$ & 48 & $14,92 \pm 0,73 b$ \\
\hline
\end{tabular}

Les moyennes d'une même colonne suivies des mêmes lettres ne sont pas significativement différentes entre elles au seuil de $5 \%$.

les témoins, aussi bien en 2015 qu'en 2016 (sauf à Maradi en 2015 où elle était quasi identique). Ainsi en 2015, le taux de larves de la MEM parasitées par $H$. hebetor était 1,5 à 4 fois plus important dans les villages de lâchers comparativement aux villages témoins. En 2016, ce taux a été 8 à 214 fois plus élevé dans les villages couverts par les lâchers que dans les villages témoins (Tab. 2).

L'enquête sur les perceptions a permis de noter que $82 \%$ des répondants ont eu connaissance de la lutte biologique pour la première fois en 2015 et $13 \%$ plus tôt, entre 2011 et 2014 . 
Tableau 2. Proportion des mines présentant des dépouilles de larves de Heliocheilus albipunctella De Joannis présentant des traces de parasitisme de Habrobracon hebetor Say par épi infesté en 2015 et 2016.

Table 2. Percentage of mines presenting dead larvae of Heliocheilus albipunctella De Joannis infected by Habrobracon hebetor Say in 2015 et 2016.

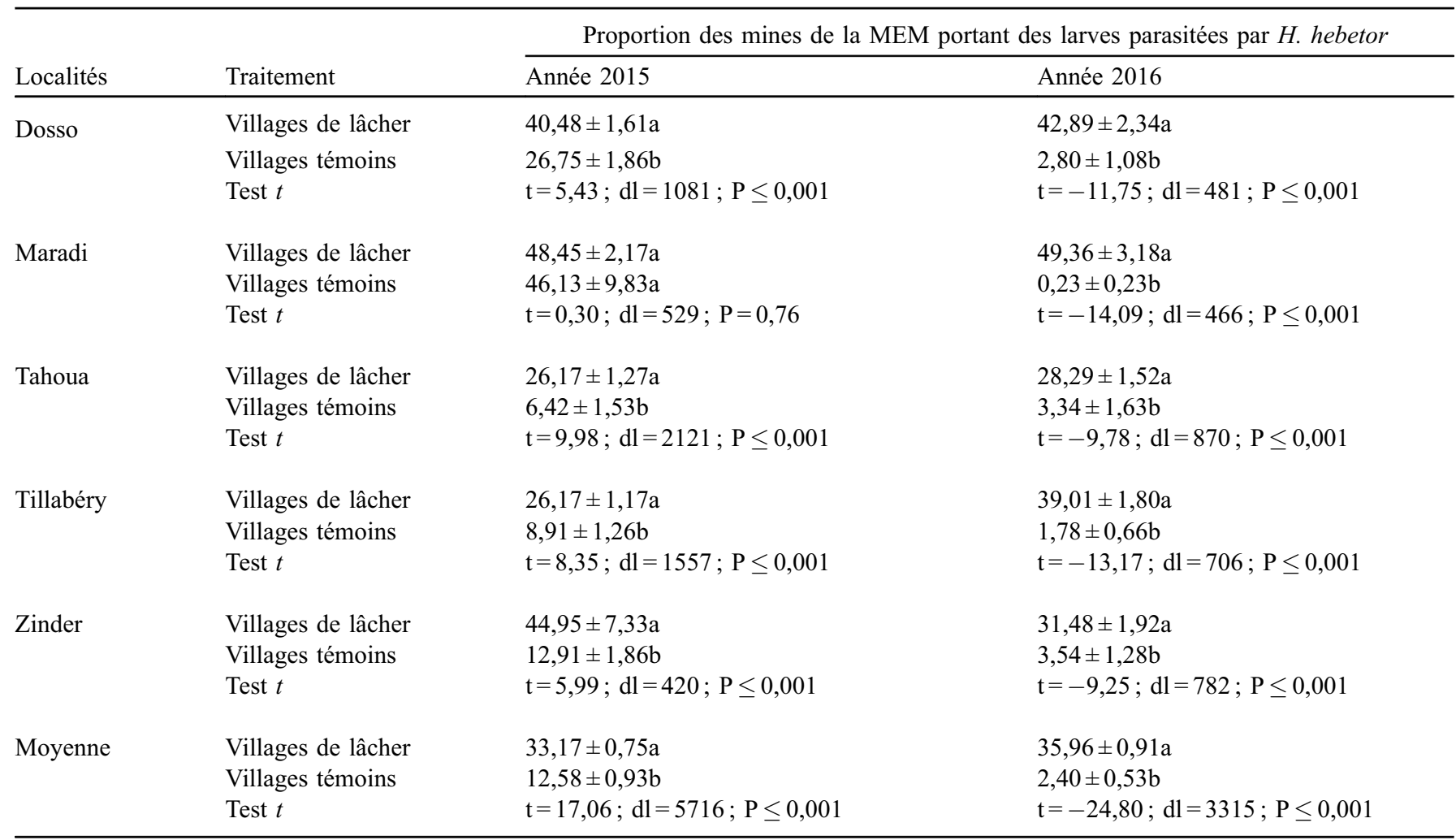

Les moyennes par localité, suivies de la même lettre ne sont pas significativement différentes entre elles au seuil de $5 \%$.

Tableau 3. Perception par les producteurs de l'opération de lutte biologique conduite en 2015 au Niger.

Table 3. Perception of farmers on biological control operations in 2015 in Niger.

\begin{tabular}{|c|c|c|c|c|c|c|}
\hline & \multicolumn{5}{|c|}{ Proportion de réponses ( $\%$ des répondants) par région } & Moyenne \\
\hline Très bonne & 3,33 & 95,05 & 70,67 & 7,18 & 42,00 & 38,66 \\
\hline Bonne & 24,67 & 2,97 & 21,33 & 40,88 & 56,00 & 31,42 \\
\hline Passable & 10,00 & 0,99 & 3,33 & 16,02 & 0,00 & 6,83 \\
\hline Médiocre & 4,00 & 0,00 & 0,00 & 2,76 & 0,00 & 1,50 \\
\hline
\end{tabular}

Par rapport à la mise en place du dispositif de lutte, $77 \%$ des répondants n'ont évoqué aucune contrainte ; $4 \%$ ont fait état du retard dans le placement du dispositif de lutte biologique, principalement dans la région de Dosso.

Les soins apportés au dispositif de lutte se résument à la protection contre la pluie pour $73 \%$ des répondants et au gardiennage des sacs pour $20 \%$ d'entre eux ; $22 \%$ n'ont mené aucune action.

Dans les cinq régions, $77 \%$ des répondants ont jugé l'opération de «très bonne» à «passable»; $7 \%$ l'ont notée «médiocre» ou «mauvaise »; $16 \%$ ne se sont pas prononcés
(Tab. 3). Dans les régions de Dosso et Tillabéry, 15\% des répondants ont noté l'opération «médiocre» ou «mauvaise» et 21 à $45 \%$ ne se sont pas prononcés.

À partir des réponses des producteurs, il a été calculé une mortalité moyenne des larves de la MEM de $72 \%$ pour l'ensemble des régions. Les taux avancés par les producteurs des régions de Maradi, Tahoua et Zinder ont été 1,4 à 1,8 fois plus importants que ceux des régions de Dosso et Tillabéry (Tab. 4).

Les producteurs des cinq régions ont estimé le gain en rendement du mil à $50 \%$ en moyenne, par rapport aux 
Tableau 4. Mortalité moyenne des larves de la MEM par épi et augmentation moyenne du rendement du mil selon les producteurs. Table 4. Average larvae mortality and average yield increase according to farmers.

\begin{tabular}{lcccc}
\hline & \multicolumn{2}{c}{ Mortalité des larves de la MEM $(\%)$} & & Augmentation de rendement du mil $(\%)$ \\
\cline { 2 - 4 } & $n$ & Moyenne \pm erreur std. & $n$ & Moyenne \pm erreur std. \\
\hline Dosso & 49 & $51,22 \pm 4,72 \mathrm{a}$ & 45 & $37,56 \pm 3,44 \mathrm{a}$ \\
Maradi & 101 & $92,97 \pm 1,70 \mathrm{~b}$ & 95 & $59,16 \pm 3,40 \mathrm{~b}$ \\
Tahoua & 147 & $90,41 \pm 1,73 \mathrm{~b}$ & 145 & $66,76 \pm 1,79 \mathrm{c}$ \\
Tillabéry & 80 & $53,00 \pm 3,69 \mathrm{a}$ & 84 & $37,86 \pm 2,23 \mathrm{a}$ \\
Zinder & 75 & $76,27 \pm 2,63 \mathrm{c}$ & 148 & $51,15 \pm 2,03 \mathrm{~b}$ \\
ANOVA & & $(\mathrm{F}=50,81 ; \mathrm{dl}=4 / 447 ; \mathrm{p}<0,001)$ & $(\mathrm{F}=24,19 ; \mathrm{dl}=4 / 512 ; \mathrm{p}<0,001)$ \\
\hline
\end{tabular}

Les moyennes d'une même colonne suivies de la même lettre ne sont pas significativement différentes entre elles au seuil de $5 \%$.

villages non couverts par la lutte biologique. Ceux de Maradi, Tahoua et Zinder ont avancé des gains en rendement de 1,4 à 1,8 fois plus importants que ceux des régions de Dosso et Tillabéry.

Le rayon d'action du dispositif de lâcher du parasitoïde $H$. hebetor a été estimé entre 2 et $8 \mathrm{~km}$ par $88 \%$ des personnes interrogées, et entre 9 et $15 \mathrm{~km}$ par $12 \%$ d'entre elles.

Le cycle du parasitoïde $H$. hebetor a été bien décrit par $16 \%$ des producteurs; $9 \%$ ont affirmé être en mesure d'identifier l'insecte dans la nature. Le parasitoïde a été jugé inoffensif pour la santé humaine par $81 \%$ des répondants. La quasi-totalité des producteurs (97\%) ont marqué leur accord pour la commercialisation des sacs de lâcher, en proposant des prix moyens de 1210 à 1810 FCFA par sac selon les régions $(1000 \mathrm{FCFA} \approx 1,5 €)$.

\section{Discussion}

En Afrique subsaharienne, la protection des végétaux est considérée comme un service public qui doit prendre en charge tous les problèmes phytosanitaires des producteurs. Mais ces dernières années, avec la raréfaction des ressources des États, les infestations croissantes des ravageurs et le désengagement des partenaires financiers de la lutte chimique en raison de ses effets négatifs sur la santé humaine et l'environnement, les moyens disponibles pour la prise en charge de la protection phytosanitaire sont limités.

Pour la gestion du problème épineux de la MEM, la démarche initiée présente quelques acquis :

- elle a permis la mise en place d'un cadre régional impliquant les producteurs, leurs organisations professionnelles, les services techniques et la recherche;

- les formations ont permis le renforcement des capacités de tous les acteurs. Dès le début de la saison pluvieuse, les zones susceptibles d'être infestées par la MEM ont été identifiées. Les déplacements dans ces zones ont permis de déterminer les besoins réels en intervention et de réaliser des économies logistiques en retenant seulement $55 \%$ des villages déclarés infestés en 2015 , et $10 \%$ en 2016 . Les besoins en sacs de lâcher ont ainsi été réduits de $33 \%$ par rapport aux estimations de début de saison;

- les séances de sensibilisation et de formation à la lutte biologique ont concerné plus de 30000 producteurs, dont au moins 3000 ont été impliqués dans la gestion du dispositif mis en place au cours des deux années.
L'approche mise en œuvre montre qu'une plus grande implication des producteurs est possible. On peut qualifier cette démarche de participative et responsabilisante. Elle rejoint celles préconisées ces dernières années par plusieurs auteurs pour le transfert des technologies agricoles (par exemple, Beaudoux et al., 1995; Singh et al., 2014; Tackie et al., 2009).

Les infestations de la MEM ont été détectées précocement et le dispositif de lutte a été placé en priorité dans les zones menacées par les ravageurs. Cette intervention basée sur l'utilisation d'organismes vivants a été préventive et correspond bien à la lutte biologique telle que définie par Ferron (1999).

Les taux d'infestation du mil par la MEM ont varié de 10 à $37 \%$ selon les régions au cours des deux années. Ils sont proches des 40 à $85 \%$ avancés par plusieurs auteurs, il y a quelques décennies (Gahukar et al., 1986; Youm et Owusu, 1998); cela montre que la MEM constitue encore une contrainte importante dans la zone agricole Sud du Niger.

L'infestation du mil a été assez hétérogène entre les régions et selon les années. Une étude effectuée au Sénégal (Soti et al., 2015) a montré que plusieurs facteurs, dont la précocité de la levée et la densité de mil, déterminent l'infestation des champs par la MEM. La variabilité notée dans la présente étude pourrait être expliquée par la répartition aléatoire de la pluviométrie et les pratiques culturales diversifiées.

La proportion des mines portant des larves de MEM parasitées a été plus importante dans les zones de lâchers du parasitoïde que dans les villages témoins. Pour les deux années, il a été noté une augmentation moyenne des taux de parasitisme de la MEM variant de 168 à $1398 \%$ consécutive au lâcher de $H$. hebetor. Le dispositif mis en place a donc contribué à la mortalité des larves de la MEM, comme déjà démontré par Ba et al. (2013) et Baoua et al. (2014).

Dans la région de Maradi, en 2015, la proportion des mines parasitées par $H$. hebetor a été comparable entre les villages témoins et ceux où les parasitoïdes ont été lâchés. Cette situation est peut-être consécutive aux lâchers répétitifs du parasitoïde qui ont concerné la région depuis 2007 (Payne et al., 2011); ces données montrent aussi la persistance du parasitoïde dans l'environnement agricole au cours des années.

Pour les deux années, les lâchers du parasitoïde ont été effectués dans moins de $3 \%$ des villages agricoles des cinq régions, mais les surfaces couvertes par le parasitoïde représentent pour chacune des années $21 \%$ des surfaces totales du mil. L'enquête sur la perception a aussi fait ressortir que plus de $80 \%$ des répondants ont expérimenté la 
technologie de lâcher de $H$. hebetor pour la première fois en 2015. Cela montre l'efficacité de la stratégie utilisée et une véritable mise à l'échelle de la technologie de lutte biologique contre la MEM au Niger.

Les réponses des producteurs ont aussi apporté les informations suivantes :

- $77 \%$ ont jugé l'opération de lutte biologique utile. Les appréciations négatives proviennent des régions de Dosso et Tillabéry, où il est noté un retard dans la mise en place des sacs en 2015 ;

- la mortalité moyenne des larves de la MEM, $76 \%$ après les lâchers, est comparable au taux de 20 à $95 \%$ noté en 2008 et 2009 par Ba et al. (2013) au Mali, au Burkina Faso et au Niger et celui de $75 \%$ rapporté par Baoua et al. (2014) dans la zone de Konni au Niger;

- l'augmentation du rendement de mil liée à la mortalité précoce des larves de la MEM a été estimée à environ 50\% par les producteurs, largement au-dessus du taux de $34 \%$ rapporté par Baoua et al. (2014). L'opération de lutte biologique a donc contribué à la réduction des pertes dues à la MEM et à un accroissement substantiel de la production ;

- le rayon d'action du dispositif de 15 sacs de lâcher a été estimé entre 2 et $8 \mathrm{~km}$ par $88 \%$ des producteurs, ce qui encadre les $5 \mathrm{~km}$ avancés par Baoua et al. (2014). Cela montre que, contrairement à la lutte chimique coûteuse, qui limite ses effets à la zone traitée et qui affecte négativement les populations d'insectes utiles, la lutte biologique avec $H$. hebetor est inoffensive pour la santé humaine et l'environnement et permet la préservation des champs de mil quel que soit le niveau de vie des producteurs;

- seuls $16 \%$ des producteurs ont pu décrire le cycle de développement du parasitoïde et $9 \%$ sa morphologie. Il s'agit là de points sur lesquels il faudra mettre l'accent lors des prochaines formations pour assurer la durabilité de la lutte biologique;

- $77 \%$ des producteurs n'ont évoqué aucune difficulté pour la gestion des sacs de lâcher. Les soins apportés concernent surtout la protection contre la pluie et le soleil;

- l'insecte parasitoïde a été jugé inoffensif pour les humains par plus de $80 \%$ des répondants. Effectivement, H. hebetor est un ectoparasite des larves de lépidoptères. Il se reproduit uniquement sur les insectes, et est donc non nuisible pour les humains ;

- la quasi-totalité des producteurs interrogés ont marqué leur disponibilité à contribuer au financement de la lutte biologique. Les prix d'achat moyens du sac proposés ne sont pas loin du prix actuel du sac de lâcher qui est de 2000 FCFA par unité $(\approx 3 €)$.

\section{Conclusion}

La démarche participative développée au cours de ces deux années a permis une lutte préventive contre la MEM. Elle a encouragé la promotion d'une méthode non chimique pour limiter les pertes dues aux ravageurs et augmenter la production du mil. Elle rejoint les résultats de Pretty et Bharucha (2015) qui ont montré que la promotion de la lutte intégrée en Afrique sub-saharienne permet la réduction de l'usage des pesticides tout en contribuant à l'intensification durable de la production agricole.

\section{Références}

Ba NM, Baoua IB, N'Diaye M, Dabire-Binso C, Sanon A, Tamo M. 2013. Biological control of the millet head miner Heliocheilus albipunctella in the Sahelian region by augmentative releases of the parasitoid wasp Habrobracon hebetor: effectiveness and farmers' perceptions. Phytoparasitica 41:569-576. DOI: 10.1007/ s12600-013-0317-x.

Baoua IB, Amadou L, Oumarou N, Payne W, Roberts JD, Stefanova K. 2014. Estimating effect of augmentative biological control on grain yields from individual pearl millet heads. J Appl Entomol 138 (4):281-288. DOI: 10.1111/jen.12077.

Beaudoux E, Bourque A, Collion MH, Delion J, Gentil D, Kabuga C. 1995. Responsabilisation des paysans en Afrique Sub-Saharienne : les meilleures pratiques. Africa Region findings; no. 33. Washington, DC: World Bank. Disponible sur http://documents. worldbank.org/curated/en/642871468223789270/Responsabilisa tion-des-paysans-en-afrique-Sub-Saharienne-les-meilleures-prati ques.

Bhatnagar VS. 1987. Conservation and encouragement of natural enemies of insect pest in dry land subsistence farming: problem, progress and prospects in the Sahelian zone. Ins Sci Appli 8: 791-795.

Bhatnagar VS. 1989. Lutte biologique contre la chenille Mineuse de l'épi du mil, Sahel PV Info 1-2: 5-8.

Ferron P. 1999. La lutte biologique : définition, concept et stratégie. La lutte biologique II, Dossier de l'environnement de l'INRA, 19: 7-17. Disponible sur http://www7.inra.fr/dpenv/ferlbd19.htm.

Gahukar RT. 1984. Insect Pests of pearl millet in West Africa. Trop Pest Manag 30(2): 142-147.

Gahukar RT, Guèvremont TH, Bhatnagar VS, Doumbia YO, Ndoye M, Pierrard G. 1986. A review of the pest status of the millet spike worm, Rhaguva albipunctella (De Joannis) (Noctuidae: Lepidoptera) and its management in the Sahel. Ins Sci Appli 7: 457-463.

INS. 2011. Profil et déterminants de la pauvreté au Niger, 38 p. Disponible sur http://www.stat-niger.org/statistique/file/Annuai res_Statistiques/Profil_Pauvrete_2011_ECVMA.pdf.

MA. 2015. Résultats définitifs de la campagne agricole 2014; perspectives alimentaires 2014-2015. Ministère de l'Agriculture. Direction des statistiques, 32 p. Disponible sur http:/www.recaniger.org/IMG/pdf/Resultats_definitifs_Campagne_agri cole_2014.pdf.

N'Doye M, Gahukar RT, Carson AG, Selvaraj C, Mbaye DF, Diallo S. 1984. Situation of plant protection problems of millet crop in Sahel. International seminar, CILSS Pest Management Project, Niamey 6-13 Dec. 1984. Comité inter-états de lutte contre la sécheresse au Sahel, Ouagadougou, Burkina Faso. Disponible sur http://pdf. usaid.gov/pdf_docs/pnady642.pdf.

Payne W, Tapsoba H, Baoua IB, Ba NM, N'Diaye M, Dabire BC. 2011. On-farm biological control of the pearl millet head miner: realization of 35 years of unsteady progress in Mali, Burkina Faso and Niger. Int J Agr Sustain 9(1): 186-193. Available from http:// www.tandfonline.com/doi/pdf/10.3763/ijas.2010.0560.

Pretty J, Bharucha ZP. 2015. Integrated pest management for sustainable intensification of agriculture in Asia and Africa. Insects 6(1): 152-182.

Sehgal D, Rajaram V, Armstead IP, Vadez V, Yadav YP, Hash CT. 2012. Integration of gene-based markers in a pearl millet genetic map for identification of candidate genes underlying drought tolerance quantitative trait loci. BMC Plt Biol12(1): 9.

Singh YP, Nayak AK, Sharma DK, Gautam RK, Singh RK, Singh R. 2014. Farmers' participatory varietal selection: A sustainable crop 
improvement approach for the 21st century. Agroecol Sust Food 38 (4): 427-444.

Soti V, Diaw M, Sow A, Thiaw I, Thiaw C, Brévault T. 2015. Effet des pratiques culturales et du contexte paysager sur l'abondance des populations de la mineuse de l'épi de mil, Heliocheilus albipunctella (Lepidoptera, Noctuidae), dans la zone de Bambey au Sénégal. Poster présenté au séminaire DIVECOSYS sur la gestion agroécologique des bioagresseurs en Afrique de l'Ouest, Dakar, Sénégal, 2 juin 2015/4 juin 2015. Disponible sur http:// agritrop.cirad.fr/577571/.
Tackie NO, Jackai LE, Ankumah R, Dingha BN, Salifu AW, Ojumu O. 2009. Integrated pest management and protection practices by limited resource farmers. J Extension 47(1): 1-9. Available from https://www.joe.org/joe/2009february/pdf/JOE_v47_1rb6.pdf.

Vercambre B. 1978. Raghuva spp. Masalia spp., chenilles des chandelles du mil en zone sahelienne. Agron Trop 33: 62-79.

Youm O, Owusu EO. 1998. Assessment of yield loss due to the millet head miner, Heliocheilus albipunctella (Lepidoptera: Noctuidae) using a damage rating scale and regression analysis in Niger. Int J Pest Manag 44: 119-121.

Citation de l'article : Amadou L, Baoua IB, Ba MN, Haussmann B, Altiné M. 2017. Gestion de la chenille mineuse de l'épi du mil par des lâchers du parasitoïde Habrobracon hebetor Say au Niger. Cah. Agric. 26: 55003. 